\title{
/CORRESPONDENCE
}

\section{Denial is Counterproductive}

To the editor:

In his October commentary (Bio/Technology 11: 1090), Bernard Dixon takes issue with the concern that I expressed in a conference paper, that advances in biotechnology face public opposition. I had documented the opposition with examples from a number of disputesover animal patenting, environmental risks, and bioengineeredfood. WithPanglossianoptimism, Dixonclaims there is a "substantial bedrock of regard for the practical achievements of science," and he describes the groups that have voiced opposition to biotechnology, such as small farmers and animal rights advocates, as "small and unrepresentative."

It is admittedly difficult to accurately measure the disenchantment with science. Public opinion polls offer varying and inconclusive data; contrary to Dixon's sources, the recent surveys I have seen indicate a troublesome degree of mistrust. Indeed, in his own column in the New Scientist (June 29, 1991) Dixon himself reported on a European Community survey that indicated such mistrust.

But surveys, I believe, tell us less about meaningful public attitudes than social movements, media messages, and governmental actions. Whatever we think about the animal rights crusades, they have surely had more than a marginal impact on science. And so too have the many disputes over environmental and health risks. As for the media, scientific fraud, environmental risks, and moral dilemmas appear to be among the most newsworthy aspects of contemporary science. Media messages both reflect and shape public attitudes, and they do not exactly convey the most comforting image of science and technology - biotechnology included - except, perhaps, on the business pages. And needless to say these days, governments seem less and less inclined to value science as an activity worth supporting.

Those of us in the field of science, technology, and society believe from our studies that the social contract that has long shaped the relationship of science to society has broken down. Public views of science are changing, and partly in response to real changes in the nature of science and its relationship to commercial goals. While Dixon tells his Bio/Technology readers to "take heart," I would advise, "take heed." Denial is counterproductive. The future of this and other scientific endeavors will rest on recognition of their social and economic consequences if scientists are to earn the public trust.

Dorothy Nelkin New York University New York, NY 10003

\section{Arm-Chair Risk Assessment \\ To the editor:}

In the clear crystal light of hindsight, a good many steps in a step-by-step approach to risk assessment might appear to be unnecessarily cautious, or even completely unnecessary. But this does not undermine the approach. On the contrary, the absence of major problems indicates that the procedure is working reasonably well. Don't be misled. Current risk assessment procedures do not represent a bureaucratic barrier standing in the way of commercial enterprise or technological innovation. It is not the environmental risk assessment that is holding up the introduction of transgenic crops.

The question to ask is where the risk assessment process would stand if a given piece of work had not been done. In the present case, our negative result made the important (if predictable) point that the act of genetic engineering does not, of itself, make any measurable difference to the ecological performance of oilseed rape. The caveats, of course, are that any extrapolation beyond the constructs we used (glufosinate and kanamycin tolerance) in one crop (oilseed rape) in these habitats (Cornwall, Berkshire, and Sutherland) in these years (1990-1992) carries with it all the uncertainties associated with any ecological prediction. But these limitations certainly don't mean that the work was not worth doing. It was one step in a step-by-step process.

Miller, Huttner, and Beachy (Bio/Technology, 11:1323, November) suggest that we should have used different (more interesting or more environmentally challenging) transgenic constructs. What actually happened was that we did not choose which transgenic constructs to use: We used all of the constructs and all of the crops that were available in sufficient quantity at the time. If more "risky" constructs like drought-tolerant perennial grasses or insect resistant-weeds had been available, then we would certainly have applied for permission to release them. It is a moot point, however, whether permission for release would ever have been given in the absence of the kind of results from less risky introductions that we have subsequently produced.

The arm-chairrisk assessments which formerly passed for ecological analysis were based on two notions: (1) it's the product, not the process that is important, and (2) genetic engineering does not alter ecological performance. While the first proposition may be eminently rational, it is quite clear that legislators didn't agree with it. Without exception, governments have applied strictures to the introduction of genetically modified organisms that were much more rigid than for the introduction of otherwise similar, nontransgenic organisms. As to the second point, the belief that a small transgenic change to genotype will have no impact on phenotype was based on the kind of in-depth analysis that goes like this: "We've thought about this very very hard, and we can't see any problems. So there isn't one."

What we did in our experimental protocol that would be capable of detecting a difference in ecological performance if one were to exist (the protocol also had the advantage of showing why any difference came about; i.e., what it was about ecological performance that had been altered; was it herbivore tolerance, growth rate, seed production, or competitive ability). So now you have two choices. You have the pundits who believed that nothing would happen, and you have the ecologists who measured it and showed that nothing happened. Who would you entrust with the protection of your environment?

Silwood Park, Ascot, U.K. SL5 7P 